\title{
Cambios en la distribución de cultivos y producciones ganaderas en España inducidos por la nueva PAC ${ }^{1}$
}

\section{Changes brought about by the new cap in the distribution of crops and livestock in Spain}

\section{Lucinio Júdez ${ }^{\star}, M^{a}$ del Rosario de Andrés ${ }^{\star \star}$, Miguel Ibáñez ${ }^{\star}$, Elvira Urzainqui ${ }^{\star \star}$ y José Luis Miguel`}

\section{INTRODUCCIÓN}

Antes de la última reforma de la Política Agraria Común (PAC) estaban vigentes las medidas contempladas en lo que se conoce con el nombre de Agenda 2000. Las ayudas concedidas a los cultivos y a los animales en un año determinado estaban directamente ligadas al número de hectáreas sembradas con los cultivos susceptibles de ayudas y al número de animales de distintas especies existentes dicho año.

La reforma propuesta en 2003 (Reglamento (CE) no 1782/2003 del Consejo) conocida con el nombre de Reforma a Medio Plazo de la PAC tiene como rasgo fundamental desvincular total o parcialmente de la superficie de los cultivos y del número de animales existentes en la explotación el montante de las ayudas percibido. Por otra parte la percepción de las ayudas implica que las explotaciones cumplan determinadas reglas

1. Este trabajo desarrolla los resultados del modelo PROMAPA que constituyen una de las bases del informe de síntesis: "Policy Recommendations", presentado a la Comisión de la UE el 31 de julio de 2007 en base al proyecto europeo GENEDEC (véase Jayet, P. A. (ed.), 2007).

* Departamento de Estadística y Métodos de Gestión en Agricultura. Escuela Técnica Superior de Ingenieros Agrónomos, Universidad Politécnica de Madrid.

** Departamento de Economía, Instituto de Economía, Geografía y Demografía, Consejo Superior de Investigaciones Científicas, Madrid. 
asociadas a buenas prácticas de cultivo y de manejo de los animales (condicionalidad).

El montante de ayudas desacopladas (pago único) que deben recibir las explotaciones de acuerdo con la nueva reforma de la PAC, es función de la media de los montantes de ayudas directas percibidas en los años 2000, 2001 y 2002. Por otro lado, una parte del total de las ayudas directas de cada explotación sobrepasando los 5.000 euros es deducida para ser dedicada a financiar programas de desarrollo rural. Esta deducción, Ilamada "modulación" de las ayudas, corresponde a un 3\% del total de ayudas en 2005, un $4 \%$ en 2006 y un $5 \%$ desde 2007 en adelante.

La aplicación de la nueva reforma de la PAC no obliga a todos los países comunitarios a aplicar el mismo sistema de desacoplamiento de ayudas. En España se ha adoptado un desacoplamiento parcial, que detallaremos más adelante, cuya aplicación ha comenzado en 2006. No obstante actualmente se está discutiendo la oportunidad de que España introduzca un esquema de desacoplamiento total de las ayudas.

Este trabajo tiene por objeto analizar los impactos, en las explotaciones tipo más afectadas por la reforma, de la implementación de las nuevas medidas de desacoplamiento para cultivos y animales tanto en el esquema de desacoplamiento parcial actual como en el hipotético caso de desacoplamiento total.

El análisis se hará a partir del modelo de Programación Matemática Positiva PROMAPA (Programación Matemática para el Análisis de Políticas Agrarias) haciendo un análisis estático comparativo, entre la situación existente en el año base 2002 en distintas explotaciones tipo, recogidas en la Red Contable Agrícola Nacional (RECAN) española, y la simulada, a partir de los resultados del modelo, una vez implementadas las medidas de la nueva PAC.

En las secciones siguientes presentaremos: el interés y reciente desarrollo de la Programación Matemática Positiva en el contexto del análisis de políticas agrarias (sección 2), una breve descripción del modelo PROMAPA (sección 3), las explotaciones tipo consideradas en el estudio (sección 4), los escenarios de política agraria del año base y del año simulado (sección 5) y la predicción de precios considerada para este último año (sección 6). Finalmente en la sección 7 analizaremos los resultados del modelo para los casos de desacoplamiento parcial y desacoplamiento total de las ayudas. Estos resultados serán comparados, por otra parte, con los que se obtendrían en el caso en que continuase la aplicación de la Agenda 2000. Las predicciones como veremos, se harán para el año 2013, en el 
que finalizan las perspectivas financieras de la UE del periodo 2002/2013 y en el que está prevista una nueva definición de la política agraria comunitaria.

\section{Programación Matemática Positiva y POlítica AgRaria}

La programación matemática y en especial la programación lineal ha constituido y todavía constituye una técnica muy utilizada en el contexto de la economía agraria. Una referencia clave en este dominio es la de Hazell y Norton (1986).

Pese a su extendida aplicación en economía agraria, la programación lineal ha sido objeto de numerosas críticas. En particular se considera que para obtener soluciones que reflejen la realidad, se precisa incluir restricciones que en la mayoría de los casos son arbitrarias.

Un procedimiento para evitar este problema lo constituye la Programación Matemática Positiva (PMP) que fue formalizada por Howit (1995), aunque se referencian aplicaciones anteriores a esta importante publicación. En síntesis, esta técnica permite a través de la estimación de los coeficientes de la función objetivo, de un modelo de programación no lineal, calibrar el modelo de modo que reproduzca la situación existente, en un año base, en la unidad modelizada (explotación, región o nación). El procedimiento de calibrado propuesto por Howit conoce un nuevo avance al introducir, para llevarlo a cabo, la maximización de la entropía (Paris y Howit, 1998).

La idoneidad de la Programación Matemática Positiva para el tratamiento y evaluación de la política agraria comun (PAC) ha impulsado recientemente un desarrollo excepcional de esta técnica como lo muestran las revisiones recientes de Heckelei y Britz (2005) y de Henry de Frahan (2005).

Sobre la base de los trabajos de Howit y Paris, se han propuesto variantes del procedimiento de calibrado tendentes a corregir algunas de sus deficiencias. Entre las propuestas mencionaremos la de Júdez et al. (1998 y 2001) donde por primera vez se propone evitar la primera fase de la PMP para efectuar el calibrado, la de Gohin y Chantreuil (1999) que presentan un procedimiento para tratar las actividades marginales, la de Helming et al. (2001) que introduce valores de las elasticidades de oferta obtenidas exogenamente, para el calibrado de los coeficientes de la función objetivo y la de Röhm y Dabber (2003) donde se propone un procedimiento para relacionar distintas 
variantes de un mismo cultivo. A estas propuestas donde el calibrado se efectúa para cada unidad modelizada con los datos de un solo año, añadiremos la de Heckelei y Wolf (2003) y la de Buysse et al. (2007) donde el calibrado se sustituye por procedimientos de estimación econométrica a partir de una serie de datos.

Simultáneamente a los desarrollos teóricos sobre el calibrado, se han efectuado recientemente un gran número de aplicaciones de la PMP para analizar los efectos de políticas agrarias (esencialmente de medidas de la política agraria común) sobre el sector agrario. Además de los trabajos anteriores que, en su mayoría, presentan junto a la propuesta de calibrado una aplicación, mencionaremos los de Arfini y Paris (1995), Heckelei y Britz (1999), Barkaoui y Butault (1999 y 2003), Röm y Dabbert (1999), Capri (2000), Paris et al. (2000), Osterburg et al. (2001), Arriaza y Gómez-Limón (2003), Júdez et al. (2004), Buysse et al. (2004), Buysse y Van Huylenbroeck (2005), Offermann et al. (2005), Blanco e Iglesias (2005), Adenauer et al. (2006) y Kuepker y Klainhauss (2006).

Señalaremos finalmente que desde hace años, varios equipos europeos están desarrollando modelos de programación matemática positiva a nivel de explotación utilizando como base de datos las Redes de Información Contable Agraria nacionales y europea. Estos modelos son utilizados con frecuencia por los responsables de las administraciones nacionales o comunitarias como instrumento de análisis de los impactos de la política agraria. Entre estos modelos mencionaremos: FARMIS (FAL- Alemania), SEPALE (Universidad de Gante, CAE de Bruselas, Universidad Católica de LovainaBélgica) y CAPRI (Universidad de Bonn-Alemania) que siendo hasta hace poco un modelo regional, ha incorporado recientemente un modelo a nivel de explotación que interacciona con un modelo de equilibrio de mercado.

El modelo PROMAPA puede situarse en la línea de investigación de los modelos antes mencionados.

\section{BREVE DESCRIPCIÓN DEL MODELO PROMAPA}

El modelo PROMAPA² es un modelo de programación matemática no lineal de explotaciones representativas, diseñado para estudiar los impactos de los cambios de medidas de políticas agrarias en el sector agrario

2. Desarrollado en el Departamento de Estadística y Métodos de Gestión en Agricultura de la ETSIA (UPM) y en el Departamento de Economía del Instituto de Economía, Geografía y Demografía (CSIC). 
español. El modelo utiliza técnicas de programación matemática positiva (PMP) para su calibrado, que puede realizarse a partir de varios procedimientos utilizando y sin utilizar informaciones a priori.

Las actividades incluidas en el modelo están constituidas por una quincena de cultivos de secano, alrededor de veinticinco cultivos de regadío, ganado bovino lechero, bovino para cría, ovino lechero y no lechero. La alimentación del ganado es endógena al modelo, pudiéndose hacer con alimentos producidos en la explotación o comprados de modo que se satisfagan las necesidades de las distintas categorías de animales en energía y proteínas y teniendo en cuenta la capacidad de ingestión. Las actividades asociadas a instrumentos de política agraria implementados en el modelo incluyen, además de la retirada de tierra obligatoria en secano y regadío, para poder percibir las ayudas directas a los cultivos COP, diversas primas para ganado (bovino lechero y de cría y ovino), el pago único, diversas ayudas directas (acopladas y desacopladas) a los cultivos, modulación de ayudas y cuotas a cultivos y animales.

Señalaremos finalmente que la fuente de datos esencial para alimentar el modelo es la RECAN, aunque también utiliza informaciones de expertos, sobre todo para los costes unitarios de los cultivos y de las distintas categorías de ganado así como para los precios asociados a la ganadería y a los alimentos comprados.

Una descripción más detallada del modelo se encuentra en Júdez et al. (2005).

\section{EXPLOTACIONES TIPO}

Las explotaciones tipo en las que se basa este estudio son las 86 explotaciones promedio que figuran en la Red Contable 2002 para las macroorientaciones técnico-económicas (MOTE) más relacionadas con la reforma de la PAC en cada una de las Comunidades Autónomas (NUTS II), con la excepción de Canarias (debido a la especificidad de las MOTES enumeradas en la RECAN para esa región). La distribución de las 86 explotaciones tipo por MOTE y Comunidad Autónoma se presentan en el cuadro 1. El conjunto de estas explotaciones representa 294.359 explotaciones a nivel nacional. 


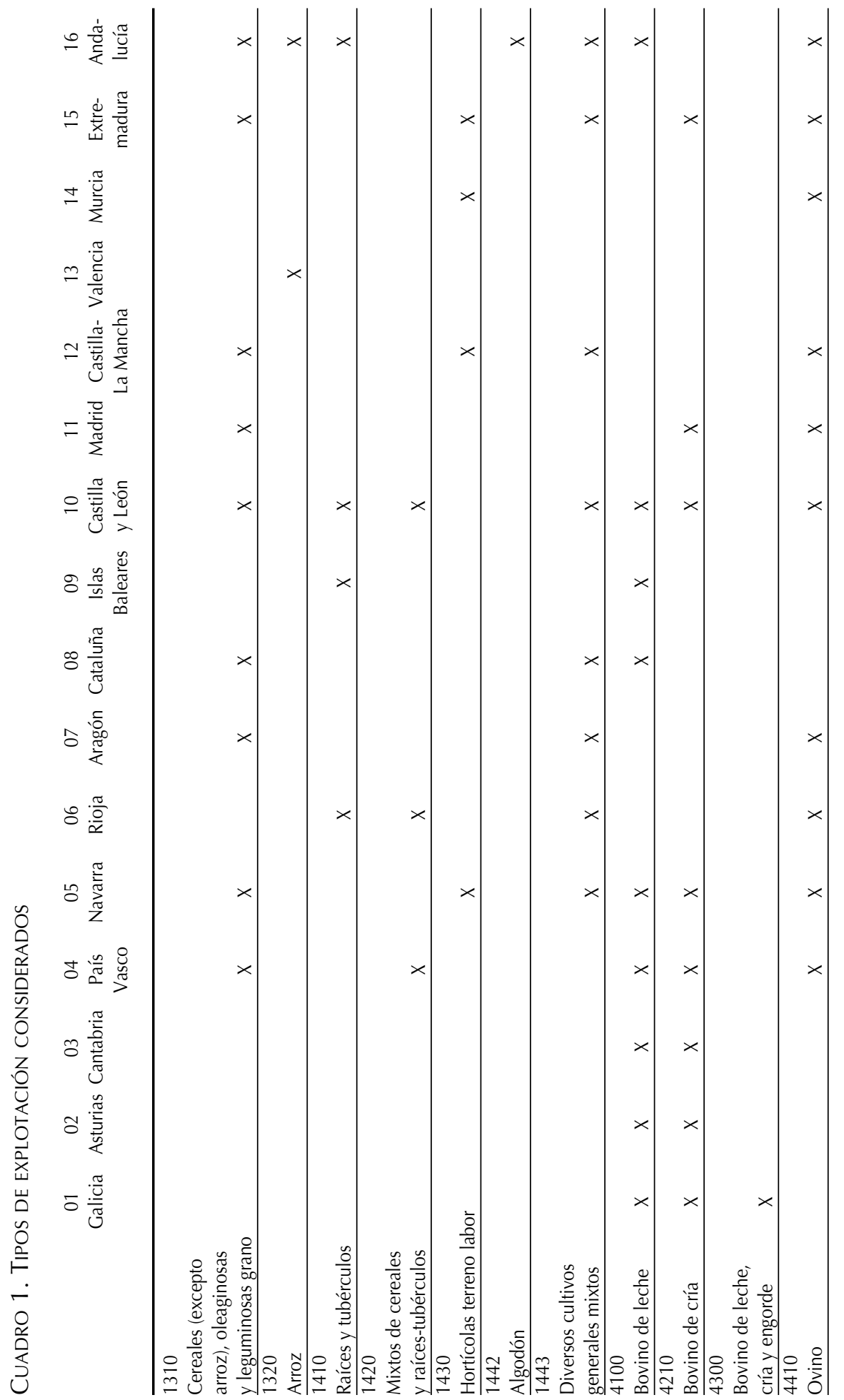




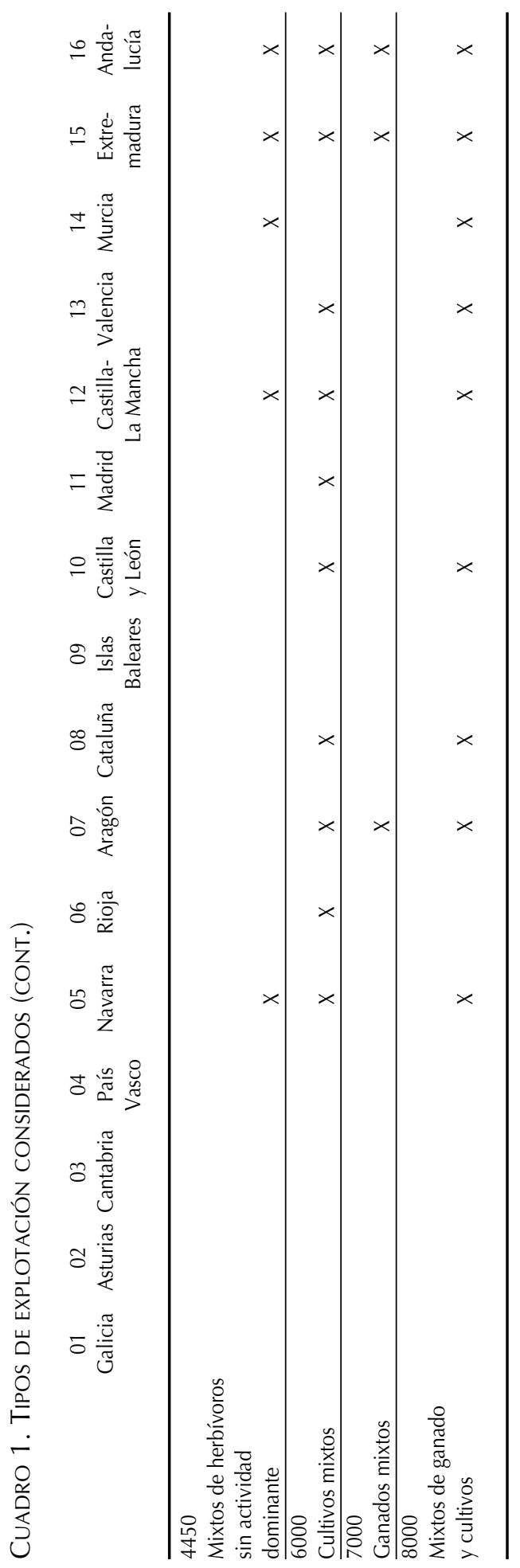

Estudios Geográficos, LXIX, 265, pp. 545-576, julio-diciembre 2008, ISSN: 0014-1496, elSSN: 1988-8546, doi: 10.3989/estgeogr.0431 


\section{ESCENARIOS}

Las medidas consideradas en el escenario de desacoplamiento parcial adoptado por España y las de la Agenda 2000 vigentes en 2002 se presentan en el cuadro 2.

Las ayudas desacopladas en el escenario de desacoplamiento total en el año simulado han sido definidas como la suma de las ayudas acopladas y desacopladas del cuadro 2, con la excepción de los siguientes pagos específicos que se han considerado acoplados: 1) prima específica para cultivos de proteaginosas; 2) prima específica a la calidad de trigo duro; 3) pago específico para el arroz; 4) pago acoplado a la superficie de algodón.

Como puede constatarse, muchas ayudas directas de los cultivos COP (Cereales, Oleaginosas y Proteaginosas) están definidas en euros por tonelada. Para determinar en estos casos la ayuda por ha, se ha multiplicado el montante de ayudas, que se definen a nivel europeo, por los rendimientos por ha referenciados, para las distintas comunidades autónomas en el Real Decreto 1893/1999 (BOE n 296 de 11 de diciembre de 1999).

Señalaremos finalmente que, tanto en el escenario de desacoplamiento parcial como en el de desacoplamiento total, se han asumido los siguientes supuestos: 1) aunque las medidas de modulación, como ya hemos visto anteriormente, se implantan de forma progresiva, en el modelo están formuladas como si se estuviese en el último período, es decir considerando una reducción del total de ayudas que supere 5.000 euros, del 5\%; 2) las hectáreas de tierra retiradas obligatoriamente son las que se retiran en el año base; 3 ) las ayudas desacopladas por explotación tipo se establecen en función de la superficie de cultivos y de los animales existentes en el año base. Es decir, el año base reemplaza al período de referencia mencionado anteriormente (media de los años naturales 2000, 2001 y 2002) en el modelo PROMAPA.

\section{PREDICCIONES DE VARIACIONES DE PRECIOS}

Las variaciones de precios que se asumen en este trabajo son las propuestas por W. Kleinhanss y B. Kuepker, basadas en las predicciones del modelo ESIM entre 2002 y 2013. Dichas predicciones, en precios reales (que corresponden a las de los precios nominales deflactadas 1,5\% anual), se muestran en el cuadro 3. 


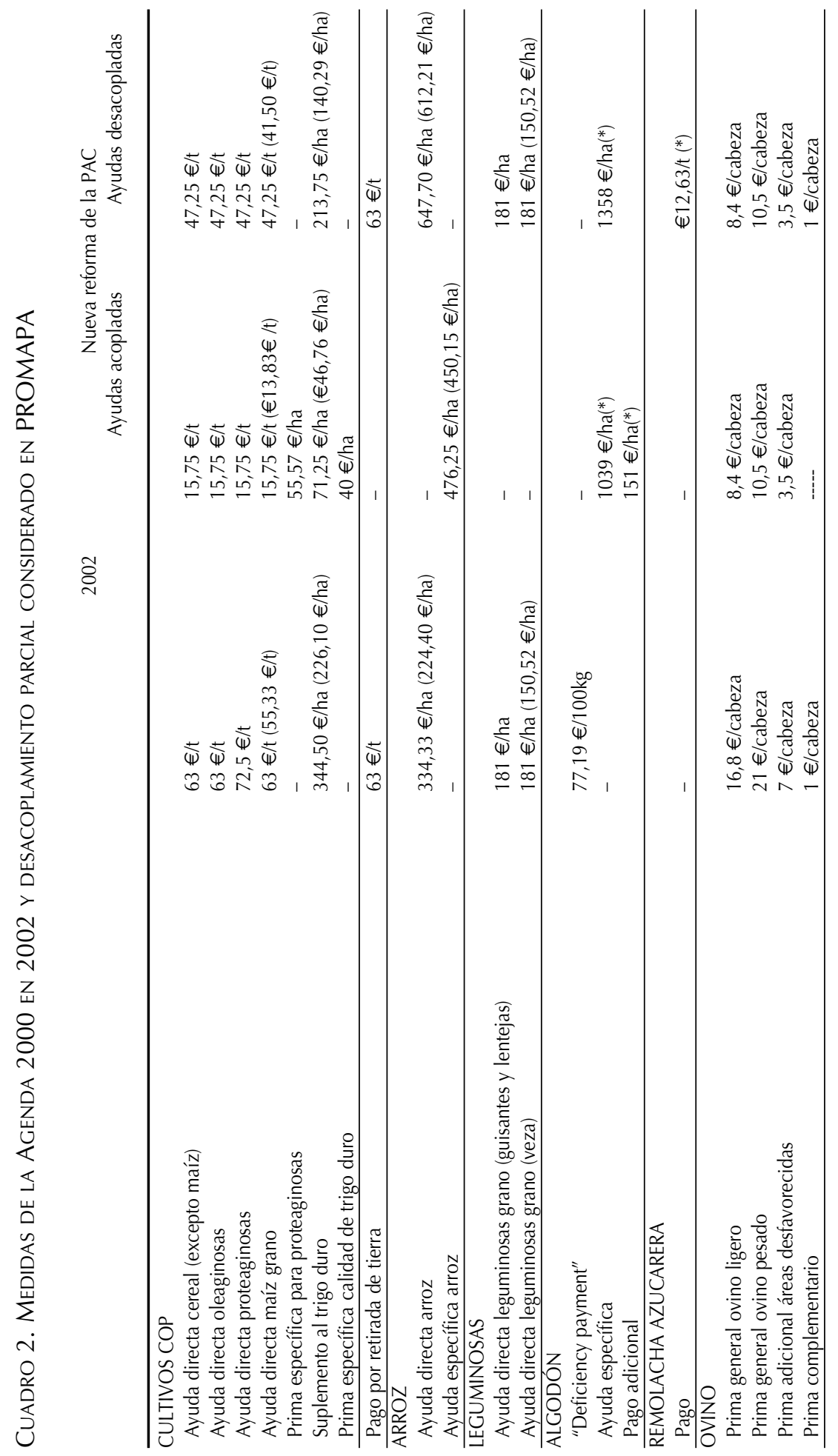




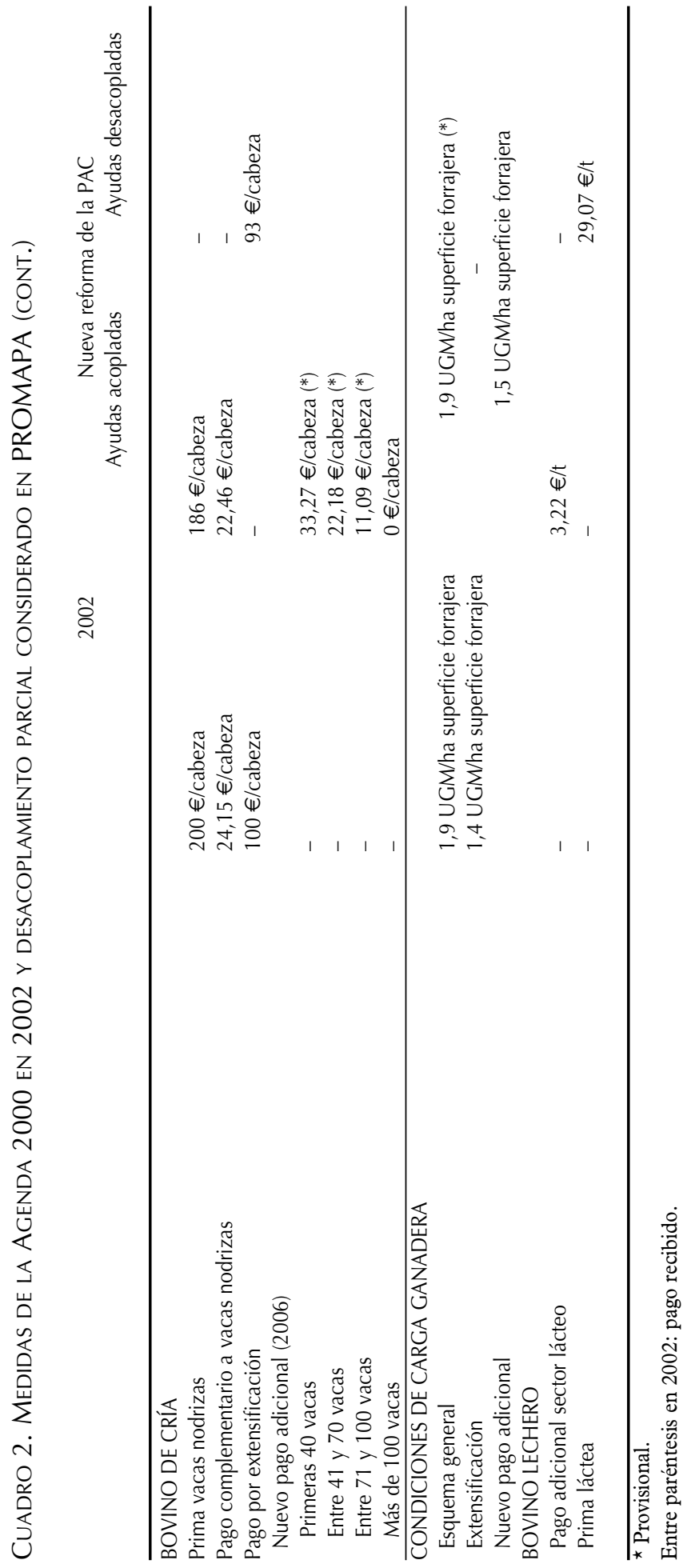


Las predicciones se han aplicado tanto a los productos vendidos como a los comprados para la alimentación del ganado. Esto hace en particular que, en nuestro caso, se produzca para los distintos escenarios simulados un descenso del coste, a veces importante, de la alimentación comprada fuera de la explotación.

Por otra parte, algunos productos introducidos en PROMAPA no tienen una predicción en el cuadro 3 (es el caso de las leguminosas, los productos hortícolas, la leche de oveja, los animales jóvenes bovinos y los corderos). Para los animales jóvenes bovinos se ha adoptado la predicción de precios del bovino y para corderos la del ovino. Para el resto de los productos no contenidos en el cuadro 3, se ha adoptado como regla general la consideración de los precios del año base.

Cuadro 3. Predicciones de variaciones en los precios Reales, en \%, ENTRE 2002 Y 2013

Agenda 2000

Desacoplamiento parcial

Desacoplamiento total

\begin{tabular}{lrrr}
\hline Trigo & $-2,0$ & 2,0 & 2,3 \\
Trigo duro & $-6,1$ & 3,5 & 3,7 \\
Cebada & $-11,9$ & $-6,1$ & $-5,7$ \\
Maíz & $-21,0$ & $-15,3$ & $-14,8$ \\
Centeno & $-14,0$ & $-14,0$ & $-14,0$ \\
Otros granos & $-21,7$ & $-16,0$ & $-15,5$ \\
Arroz & $-48,4$ & $-48,4$ & $-48,4$ \\
Remolacha azucarera & $-27,6$ & $-25,0$ & $-24,9$ \\
Patatas & $-43,9$ & $-38,0$ & $-37,7$ \\
Girasol & $-7,4$ & $-5,2$ & $-5,0$ \\
Forraje & $-24,7$ & $-48,0$ & $-46,2$ \\
Pasto & $-28,0$ & $-44,8$ & $-42,5$ \\
Leche & $-19,0$ & $-22,8$ & $-22,4$ \\
Bovino & $-18,4$ & $-8,8$ & $-4,6$ \\
Ovino & 4,1 & 31,0 & 37,6 \\
\hline
\end{tabular}

Fuente: Comunicación de W. Kleinhanss y B. Kuepker, basada en los resultados del modelo ESIM.

\section{ANÁLISIS DE RESULTADOS}

Los resultados se han obtenido con el software GAMS/SBB, calibrando el modelo con elasticidades de oferta exógena (Helming et al., 2001), considerando las elasticidades utilizadas por FAL en el modelo FARMIS, salvo para los productos hortícolas, para los que hemos supuesto una elasticidad del 0,1 de acuerdo con el trabajo de Ibáñez y Pérez, (1999). 
En las subsecciones siguientes analizaremos la influencia de las distintas políticas agrarias sobre los resultados, tanto a nivel global como a nivel regional cuando sea necesario explicar las diferentes variaciones que se observan entre las Comunidades Autónomas. El análisis de los resultados agregando los resultados de las 86 explotaciones tipo se presentan en el cuadro 4. Para proceder a la agregación, se han multiplicado los resultados obtenidos para cada explotación tipo por el número de explotaciones que representan según la RECAN.

En primer lugar se analizarán los resultados obtenidos para los cultivos, a continuación examinaremos los relativos al ganado y finalmente estudiaremos los resultados económicos.

\section{Resultados por grupos de cultivos}

1. El descenso de precios, que en todos los escenarios experimentan la mayoría de los cultivos considerados, Ileva consigo un descenso de la actividad agraria respecto al año base, que se traduce, incluso en el supuesto de continuar las medidas de la Agenda 2000 sin desacoplamiento, en una disminución de la superficie utilizada superior al 0,5\%. Con las políticas de desacoplamiento, lógicamente, esta disminución es mayor, siendo de más del $2 \%$ en el caso de desacoplamiento parcial y superando el $5 \%$ con el escenario de desacoplamiento total.

\section{GRÁFICO 1. VARIACIÓN DE LA SUPERFICIE CULTIVADA POR GRUPOS DE CULTIVOS}

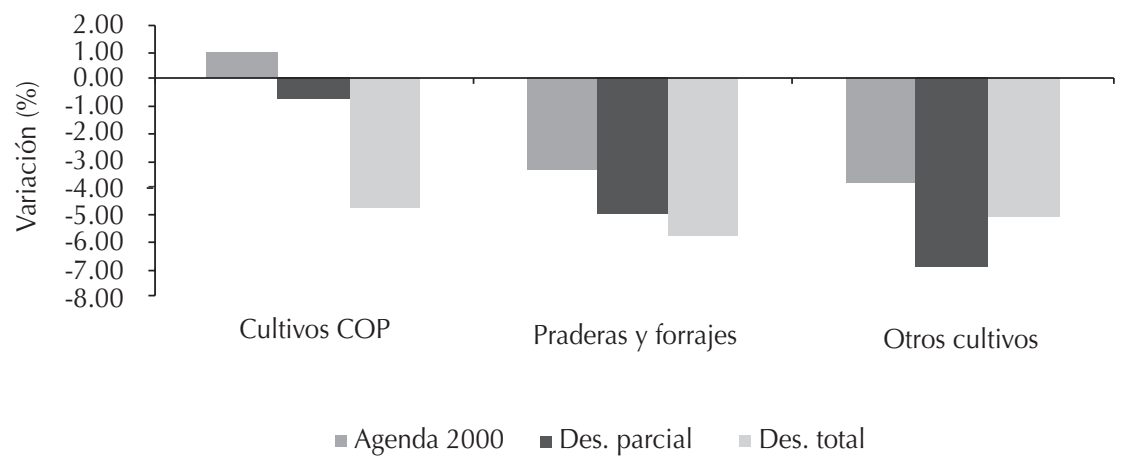

2. Observando la evolución de los grupos de cultivos en los escenarios de desacoplamiento (parcial y total) constatamos que el descenso de superficie de los cultivos COP y de las praderas y cultivos forrajeros, que están 
afectados por la política de desacoplamiento, es tanto mayor cuanto mayor es el grado de desacoplamiento. Esto no sucede en el caso de los "otros cultivos" que, como veremos, es un grupo heterogéneo cuya evolución depende menos de la política de desacoplamiento. Para estos cultivos el descenso es menor con los escenarios de desacoplamiento total, debido a que los cultivos que pueden sustituirlos (esencialmente los cultivos COP) tienen ingresos acoplados por ha superiores en el caso de un desacoplamiento parcial.

Cuadro 4. Resultados agregados de las 86 explotaciones tipo. Explotaciones REPRESENTADAS: 294.359

$\begin{array}{cccc}\begin{array}{c}\text { Año base } \\ 2002\end{array} & \text { Agenda } 2000 & \begin{array}{c}\text { Desacoplamiento } \\ \text { parcial }\end{array} & \begin{array}{c}\text { Desacoplamiento } \\ \text { total }\end{array} \\ & \text { Valor Variación } & \text { Valor Variación } & \text { Valor Variación }\end{array}$
(\%)
$(\%)$
(\%)

\begin{tabular}{|c|c|c|c|c|c|c|c|}
\hline \multicolumn{8}{|l|}{ CULTIVOS } \\
\hline Garbanzos secano (miles ha) & 25,37 & 28,57 & 12,62 & 26,59 & 4,81 & 29,2 & 15,12 \\
\hline Arroz (miles ha) & 28,89 & 26,22 & $-9,22$ & 28,35 & $-1,86$ & 28,62 & $-0,91$ \\
\hline Remolacha azucarera regadío (miles ha) & 96,02 & 72,81 & $-24,18$ & 83,25 & $-13,3$ & 84,1 & $-12,42$ \\
\hline Algodón regadío (miles ha) & 89,92 & 96,57 & 7,4 & 80,81 & $-10,14$ & 81,19 & $-9,71$ \\
\hline Pimiento pimentón regadío(miles ha) & 2,32 & 2,34 & 0,59 & 2,34 & 0,58 & 2,34 & 0,59 \\
\hline Patata temprana regadío (miles ha) & 8,32 & 7,03 & $-15,44$ & 7,35 & $-11,63$ & 7,36 & $-11,52$ \\
\hline Patata media estación regadío (miles ha) & 12,29 & 10,67 & $-13,19$ & 11,18 & $-9,07$ & 11,2 & $-8,88$ \\
\hline Patata tardía regadío (miles ha) & 14,54 & 13,06 & $-10,14$ & 13,49 & $-7,23$ & 13,54 & $-6,84$ \\
\hline Espárragos regadío, (miles ha) & 0,54 & 0,55 & 1,57 & 0,55 & 1,42 & 0,55 & 1,47 \\
\hline Melón regadío (miles ha) & 1,6 & 1,62 & 0,77 & 1,62 & 1,04 & 1,62 & 1,21 \\
\hline Tomate regadío (miles ha) & 16,51 & 16,61 & 0,63 & 16,57 & 0,39 & 16,58 & 0,41 \\
\hline Pimiento regadío (miles ha) & 0,2 & 0,2 & 0,41 & 0,2 & 0,36 & 0,2 & 0,34 \\
\hline Alcachofa regadío (miles ha) & 3,61 & 3,64 & 0,79 & 3,63 & 0,49 & 3,63 & 0,48 \\
\hline Coliflor regadío (miles ha) & 0,53 & 0,53 & 0,7 & 0,53 & 0,57 & 0,53 & 0,55 \\
\hline Ajo regadío (miles ha) & 10,95 & 10,97 & 0,24 & 10,99 & 0,37 & 10,99 & 0,39 \\
\hline Cebolla regadío (miles ha) & 2,47 & 2,48 & 0,43 & 2,48 & 0,71 & 2,48 & 0,7 \\
\hline Judía verde regadío (miles ha) & 0,31 & 0,4 & 28,03 & 0,39 & 23,63 & 0,39 & 24,13 \\
\hline Guisante seco regadío (miles ha) & 1,91 & 2,16 & 12,98 & 2,03 & 6,42 & 1,99 & 4,12 \\
\hline Trigo duro secano (miles ha) & 355,44 & 359,55 & 1,16 & 282,48 & $-20,53$ & 248,07 & $-30,21$ \\
\hline Trigo duro regadío (miles ha) & 47,26 & 49,62 & 5 & 47,39 & 0,28 & 46,18 & $-2,29$ \\
\hline Trigo blando secano (miles ha) & 897,36 & 943,14 & 5,1 & 944,45 & 5,25 & 904,46 & 0,79 \\
\hline Trigo blando regadío (miles ha) & 120,86 & 141,61 & 17,17 & 149,13 & 23,39 & 148,28 & 22,69 \\
\hline Centeno secano (miles ha) & 50,72 & 49,66 & $-2,08$ & 47,67 & $-6,01$ & 45,2 & $-10,88$ \\
\hline Centeno regadío (miles ha) & 3,9 & 4,13 & 5,85 & 3,99 & 2,27 & 3,91 & 0,21 \\
\hline Cebada secano (miles ha) & $2.882,61$ & $2.835,2$ & $-1,64$ & $2.861,11$ & $-0,75$ & $2.764,17$ & $-4,11$ \\
\hline Cebada regadío (miles ha) & 188,51 & 218,77 & 16,05 & 227,42 & 20,64 & 223,04 & 18,32 \\
\hline Avena secano (miles ha) & 157,24 & 142,65 & $-9,28$ & 142,47 & $-9,39$ & 140,39 & $-10,72$ \\
\hline Avena regadío (miles ha) & 0,55 & 0,56 & 1,1 & 0,58 & 5,84 & 0,57 & 2,93 \\
\hline Maíz grano secano (miles ha) & 2,73 & 2,87 & 5,21 & 2,87 & 5,15 & 2,62 & $-3,87$ \\
\hline Maíz grano regadío (miles ha) & 374,37 & 341,23 & $-8,85$ & 331,91 & $-11,34$ & 320,64 & $-14,35$ \\
\hline
\end{tabular}


558 • LUCINIO JÚDEZ, M. a DEL ROSARIO DE ANDRÉS, MIGUEL IBÁÑEZ, ELVIRA URZAINQUI Y JOSÉ LUIS MIGUEL

\section{Cuadro 4. Resultados agregados de las 86 explotaciones tipo. EXPlotaciones REPRESENTADAS: 294.359 (CONT.)}

$\begin{array}{cccc}\begin{array}{c}\text { Año base } \\ 2002\end{array} & \text { Agenda } 2000 & \begin{array}{c}\text { Desacoplamiento } \\ \text { parcial }\end{array} & \begin{array}{c}\text { Desacoplamiento } \\ \text { total }\end{array} \\ & \text { Valor Variación } & \text { Valor Variación } & \text { Valor Variación }\end{array}$

$(\%) \quad(\%)$

\begin{tabular}{|c|c|c|c|c|c|c|c|}
\hline Girasol secano (miles ha) & 496,41 & 518,69 & 4,49 & 476,18 & $-4,08$ & 447,11 & $-9,93$ \\
\hline Girasol regadío (miles ha) & 103,82 & 123,67 & 19,12 & 115,75 & 11,49 & 111,88 & 7,76 \\
\hline Veza secano (miles ha) & 34,16 & 40,64 & 18,97 & 33,69 & $-1,36$ & 35,91 & 5,14 \\
\hline Alfalfa secano (miles ha) & 158,21 & 137,82 & $-12,89$ & 117,78 & $-25,56$ & 126,04 & $-20,34$ \\
\hline Alfalfa regadío (miles ha) & 138,22 & 116,86 & $-15,46$ & 88,26 & $-36,15$ & 94 & -32 \\
\hline Cereales invierno forraje secano (miles ha) & 3,16 & 2,87 & $-9,38$ & 3,17 & 0,03 & 3,17 & 0,03 \\
\hline Maíz forraje secano (miles ha) & 2,17 & 1,44 & $-33,65$ & 1,72 & $-20,81$ & 1,7 & $-21,69$ \\
\hline Maíz forraje regadío (miles ha) & 8,03 & 7,16 & $-10,94$ & 7,73 & $-3,74$ & 7,86 & $-2,16$ \\
\hline Pradera temporal secano (miles ha) & 247,03 & 236,6 & $-4,22$ & 240,03 & $-2,83$ & 245,16 & $-0,76$ \\
\hline Pradera temporal regadío (miles ha) & 21,89 & 22,17 & 1,3 & 22,35 & 2,11 & 22,34 & 2,06 \\
\hline Pradera permanente secano (miles ha) & $1.817,11$ & $1.817,11$ & 0 & $1.817,11$ & 0 & $1.817,11$ & 0 \\
\hline Pradera permanente regadío (miles ha) & 23,32 & 23,32 & 0 & 23,32 & 0 & 23,32 & 0 \\
\hline \multicolumn{8}{|l|}{ GANADERÍA } \\
\hline Vaca nodriza (miles cabezas) & 961,35 & 960,8 & $-0,06$ & 926,36 & $-3,64$ & 858,51 & $-10,7$ \\
\hline Vaca lechera (miles cabezas) & 999,59 & 943,3 & $-5,63$ & 969,24 & $-3,04$ & 961,19 & $-3,84$ \\
\hline Oveja (miles cabezas) & $13.815,66$ & $13.879,23$ & 0,46 & $14.376,39$ & 4,06 & $13.861,73$ & 0,33 \\
\hline UGM (miles UGM) & $4.348,58$ & $4.289,94$ & $-1,35$ & $4.357,07$ & 0,2 & $4.194,22$ & $-3,55$ \\
\hline \multicolumn{8}{|l|}{ SUPERFICIE NO UTILIZADA } \\
\hline Superficie no utilizada secano (miles ha) & 0 & 41,25 & $\operatorname{lnf}$ & 157,33 & Inf & 380,33 & Inf \\
\hline Superficie no utilizada regadío (miles ha) & 0 & 0,19 & $\operatorname{lnf}$ & 38,09 & $\operatorname{lnf}$ & 54,38 & $\operatorname{lnf}$ \\
\hline \multicolumn{8}{|l|}{ SUPERFICIE UTILIZADA (RESUMEN) } \\
\hline Otros cultivos secano (miles ha) & 59,52 & 69,21 & 16,26 & 60,28 & 1,27 & 65,11 & 9,39 \\
\hline Total cultivos COP secano (miles ha) & $4.842,51$ & $4.851,75$ & 0,19 & $4.757,23$ & $-1,76$ & $4.552,02$ & -6 \\
\hline \multicolumn{8}{|l|}{ Total praderas y cultivos forrajeros } \\
\hline secano (miles ha) & $2.227,69$ & $2.166,24$ & $-2,76$ & $2.154,88$ & $-3,27$ & $2.132,26$ & $-4,28$ \\
\hline Otros cultivos regadío (miles ha) & 289,03 & 265,72 & $-8,07$ & 263,73 & $-8,75$ & 265,32 & $-8,2$ \\
\hline Total cultivos COP regadío (miles ha) & 841,18 & 881,76 & 4,82 & 878,2 & 4,4 & 856,47 & 1,82 \\
\hline $\begin{array}{l}\text { Total praderas y cultivos forrajeros } \\
\text { regadío (miles ha) } \\
\end{array}$ & 191,47 & 169,5 & $-11,47$ & 141,67 & $-26,01$ & 145,5 & $-24,01$ \\
\hline \multicolumn{8}{|l|}{ RESULTADOS ECONÓMICOS } \\
\hline Ayudas acopladas (millones $€$ ) & $1.977,75$ & $2.005,15$ & 1,39 & 735,03 & $-62,84$ & 121,39 & $-93,86$ \\
\hline Ayudas desacopladas (millones $€$ ) & 0 & 0 & 0 & $1.551,27$ & $\operatorname{lnf}$ & $2.162,48$ & Inf \\
\hline $\begin{array}{l}\text { Ayudas totales antes modulación } \\
\text { (millones } € \text { ) }\end{array}$ & $1.977,75$ & $2.005,15$ & 1,39 & $2.286,3$ & 15,6 & $2.283,87$ & 15,48 \\
\hline $\begin{array}{l}\text { Reducción por modulación } \\
\text { (millones } € \text { ) }\end{array}$ & 0 & 0 & 0 & 50,7 & Inf & 50,68 & $\operatorname{lnf}$ \\
\hline $\begin{array}{l}\text { Ayudas totales tras modulación } \\
\text { (millones } € \text { ) }\end{array}$ & $1.977,75$ & $2.005,15$ & 1,39 & $2.235,59$ & 13,04 & $2.233,19$ & 12,92 \\
\hline $\begin{array}{l}\text { Margen total tras modulación } \\
\text { (millones } € \text { ) }\end{array}$ & $6.359,78$ & $5.580,3$ & $-12,26$ & $6.281,78$ & $-1,23$ & $6.298,65$ & $-0,96$ \\
\hline$\%$ ayudas / margen & 31,1 & 35,93 & & 35,59 & & 35,45 & \\
\hline
\end{tabular}


En los gráficos 2 a 4 se representan las variaciones de las superficies de los cultivos COP de regadío y de secano. A partir de estos gráficos haremos los siguientes comentarios.

1. Considerando únicamente los escenarios de desacoplamiento parcial y total se constata que:

- Debido al efecto de desacoplamiento de los pagos directos a los cultivos, los incrementos de superficie cultivada son más débiles y las disminuciones más fuertes cuanto mayor es el grado de desacoplamiento.

- Las pérdidas de ingresos acoplados en los distintos escenarios, debida a las predicciones de precios y al desacoplamiento, que se muestran en el cuadro $5^{3}$, constituye un elemento clave para explicar la evolución de los cultivos COP en secano y regadío de los gráficos 3 y 4.

Cuadro 5. Pérdida media (en euros), ResPeCto del año base, de lOS INGResos ACOPLADOS POR HA DE CULTIVOS COP CON ESCENARIOS DE DESACOPLAMIENTO

Desacoplamiento parcial Desacoplamiento total

\begin{tabular}{lll}
\hline Trigo de secano & 123,12 & 159,86 \\
Cebada de secano & 147,39 & 184,06 \\
Girasol de secano & 144,07 & 181,33 \\
Trigo duro de secano & 259,66 & 343,66 \\
\hline Trigo de regadío & 204,76 & 262,82 \\
Cebada de regadío & 242,57 & 300,72 \\
Girasol de regadío & 312,60 & 395,11 \\
Trigo duro de regadío & 409,80 & 539,06 \\
Maíz de regadío & 541,76 & 635,87 \\
\hline
\end{tabular}

- Pese a que las reducciones de los cultivos en regadío son superiores a las de secano, se observan mayores incrementos de las superficies de cultivos COP en la primera categoría de tierra. Esto es debido a que en regadío compiten con otros cultivos más penalizados que ellos por el precio (remolacha azucarera, patatas y alfalfa) o por el desacoplamiento (caso del algodón, cuyo montante acoplado es casi la mitad de lo que percibía el año base con el sistema de "deficiency payment").

3. Dicho cuadro se ha elaborado con datos de la explotación tipo media del conjunto de España. 


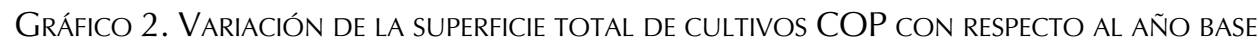

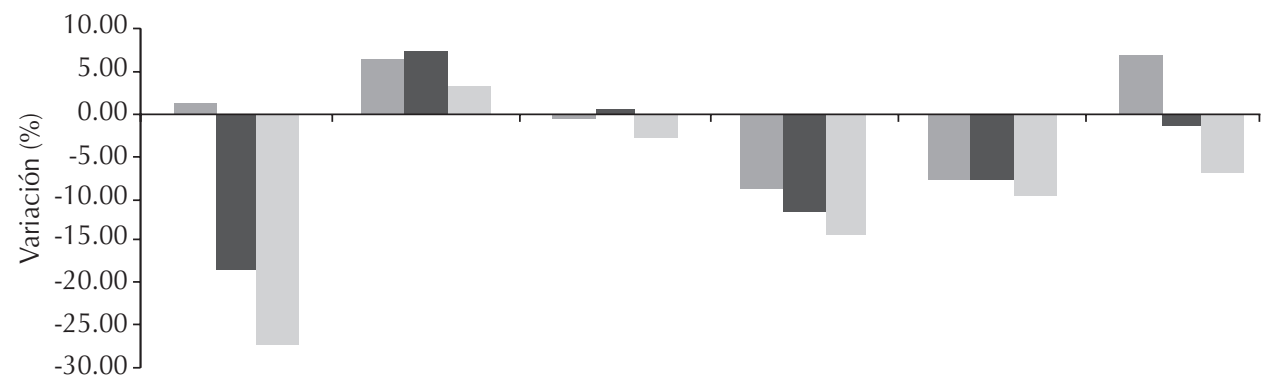

Trigo duro Trigo Maíz grano Otros cereales Girasol

- Agenda 2000 - Des. parcial $\square$ Des. Total

Gráfico 3. Variación de LA SUPEFICIE DE CULtivos COP DE REGAdíO

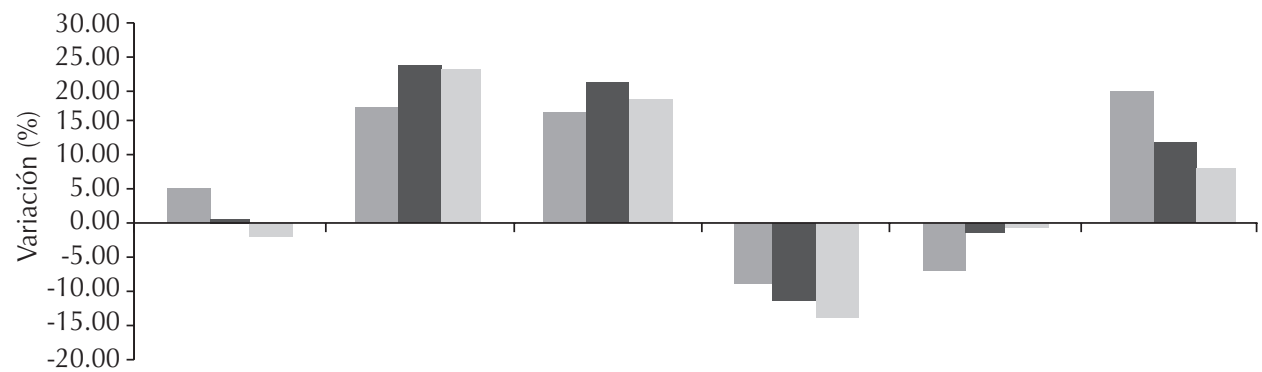

Trigo duro
regadío Trigo regadío Cebada regadío Maíz regadío $\begin{gathered}\text { Otros cereales Girasol regadío } \\ \text { regadío }\end{gathered}$ - Agenda 2000 - Des. parcial $\unrhd$ Des. total

GrÁfico 4. VARIACIÓn de LA SUPERfiCie de CULtivos COP DE SECANO

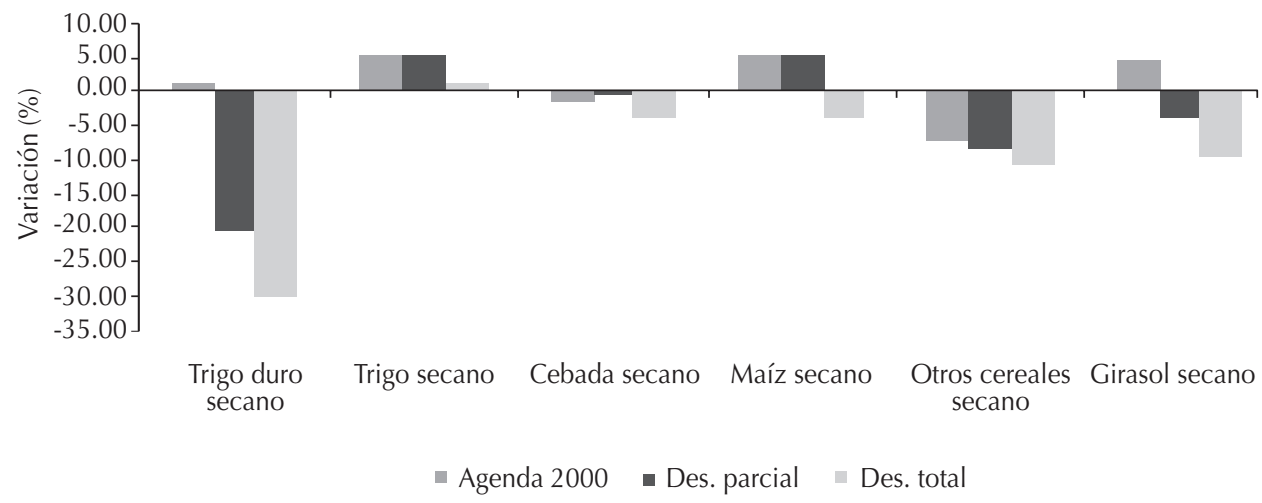


- En secano sólo el cultivo menos penalizado del cuadro 5 (trigo) incrementa su superficie. También lo hace el maíz con el escenario de desacoplamiento parcial; este caso, sin embargo, es muy particular, pues tiene una superficie muy reducida y concentrada únicamente en Galicia, donde su cultivo tiene lugar junto con las praderas temporales y permanentes.

- En regadío incrementan su superficie, sustituyendo a otros cultivos, COP y no COP, los tres cultivos menos penalizados por el efecto combinado de los precios y el desacoplamiento (trigo, cebada y girasol).

2. Bajo la hipótesis de continuación de las medidas de la Agenda 2000, la pérdida de ingresos acoplados por ha se debe exclusivamente a la reducción de precios y se muestra en el cuadro 64 .

Cuadro 6. Pérdida media (en euros), Respecto del año base, de los ingresos ACOPLADOS POR HA DE LOS CULtivos COP en EL ESCENARIO DE LA AGENDA 2000

\begin{tabular}{lcc} 
& Secano & Regadío \\
\hline Trigo & 7,08 & 11,89 \\
Trigo duro & 17,17 & 29,70 \\
Girasol & 19,73 & 35,68 \\
Cebada & 33,53 & 50,56 \\
Maíz & - & 276,49 \\
\hline
\end{tabular}

Aunque, como en el caso anterior, no existe una relación inversa exacta en el sentido de que a menor pérdida media mayor incremento del culti$\mathrm{vo}^{5}$, se observa que son los cultivos con menores pérdidas los que se incrementan, tanto en secano (trigo duro y girasol) como en regadío (a los cultivos anteriores se añade la cebada). Los mayores incrementos en regadío se deben a las mismas causas que se indicaron para los escenarios de desacoplamiento.

4. Como el cuadro 5, este cuadro se ha elaborado con datos de la explotación tipo media del conjunto de España.

5. Esto es debido a que los datos de los cuadro 5 y cuadro 6 son datos medios y en cada tipo de las 86 explotaciones tipo consideradas en este trabajo, los cultivos tienen distintos rendimientos y precios y compiten con cultivos diferentes. 
Variaciones de las praderas y los cultivos forrajeros

Para analizar la evolución de este grupo de cultivos es preciso estudiar, por una parte, el cultivo forrajero más importante: la alfalfa, y por otra el resto que está constituido esencialmente por praderas temporales y permanentes (también están incluidos el maíz forrajero y los cereales de invierno para alimento del ganado, aunque su superficie representa menos del $1 \%$ del total). Mientras la alfalfa puede ser utilizada como alimento del ganado en la propia explotación o vendida, el modelo considera que los demás cultivos de este grupo sólo pueden ser utilizados para alimentación del ganado.

El gráfico 5 muestra la variación de la superficie de estos dos subgrupos. La alfalfa está en su mayor parte dedicada a la venta, por lo que su evolución es función de su precio y de los ingresos por ha de los cultivos que compiten con ella, que son esencialmente los cultivos COP. En todos los escenarios, la alfalfa es menos rentable que estos productos. Por otra parte, la disminución de su precio en los escenarios de desacoplamiento es considerable (más del 45\% según ESIM) siendo prácticamente el doble que en el supuesto de continuar la Agenda 2000. En consecuencia, el descenso de la superficie de alfalfa es mayor cuando se asumen los escenarios de desacoplamiento. En estos, la mayor disminución corresponde al desacoplamiento parcial, donde los cultivos COP producen mayores ingresos acoplados por ha que en el caso de desacoplamiento total.

La variación del resto de los cultivos forrajeros está íntimamente ligada a la evolución del ganado (esencialmente vacuno), que estudiaremos posteriormente.

GRÁFICO 5. VARIACIÓN DE LA SUPERFICIE DE PRADERAS Y CUTIVOS FORRAJEROS RESPECTO AL AÑO BASE

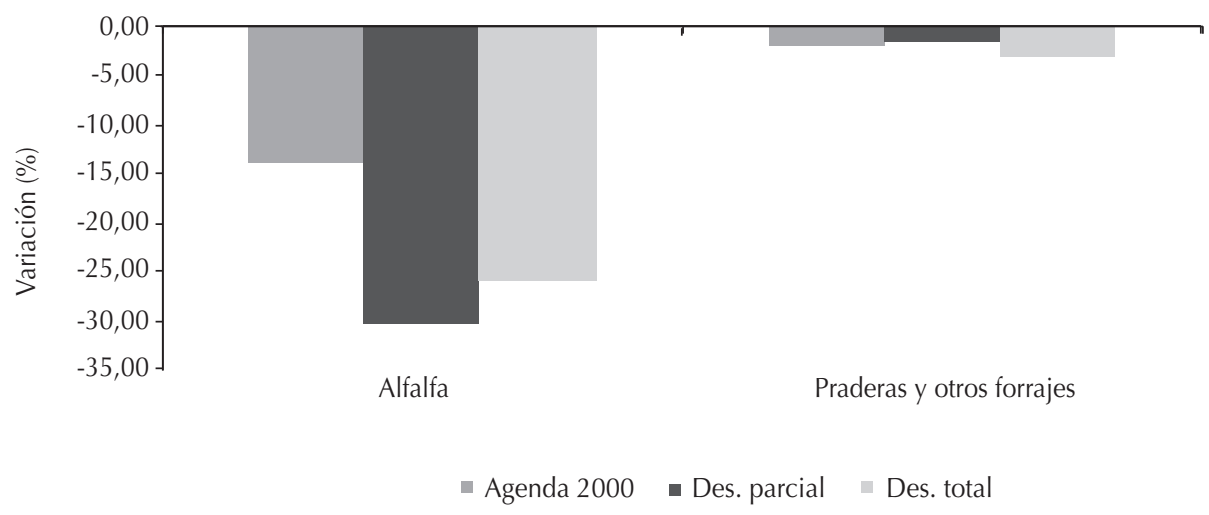


La formulación hecha en PROMAPA permite distinguir entre la superficie existente y la utilizada. El hecho de que no se utilice toda la superficie de praderas existente puede deberse al interés en conservarla para preservar una "carga ganadera" suficientemente baja que dé derecho a primas adicionales para el ganado, o al hecho de que no existan otros cultivos para su sustitución. En este trabajo, el porcentaje de superficie forrajera existente no utilizada es de 1,25\% en el supuesto de continuación de la Agenda 2000, de alrededor del $1 \%$ en el caso de desacoplamiento parcial y de más del $2,5 \%$ en el caso del desacoplamiento total

Variación de otros cultivos (no COP y no forrajeros)

Las leguminosas para grano (guisantes y veza), algodón, arroz, remolacha azucarera y patata representan más del $85 \%$ de los cultivos no COP si se exceptúan los cultivos forrajeros vistos anteriormente.

Las variaciones de estos cultivos con las distintas políticas agrarias consideradas se presentan en el gráfico 6.

GRÁFICO 6. VARIACIÓN DE LA SUPERFICIE DE CUTIVOS NO COP NO FORRAJEROS RESPECTO AL AÑO BASE

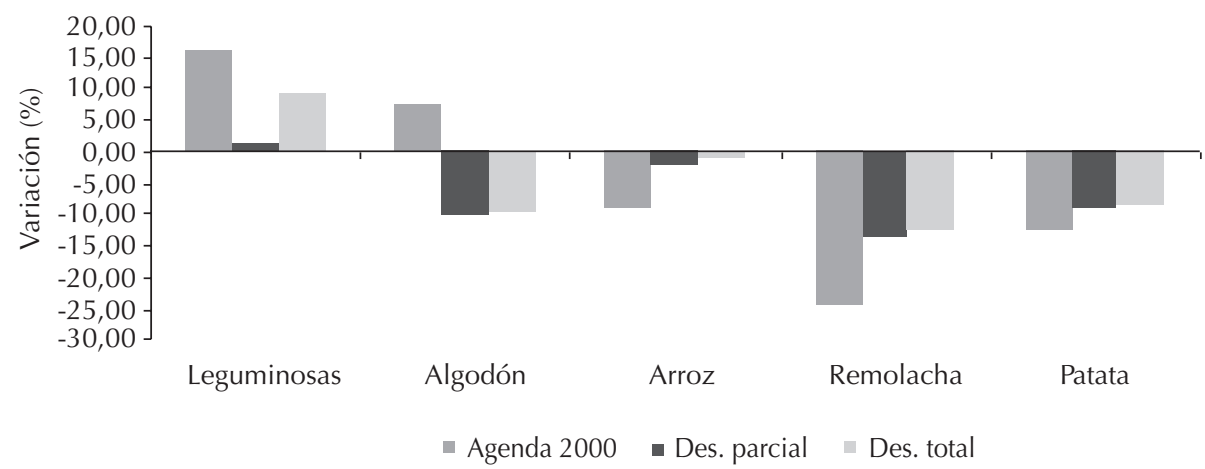

Todos estos cultivos son de regadío salvo las leguminosas. Sus variaciones, como puede observarse, dependen del escenario de política agraria considerado.

Para las leguminosas hemos asumido precios constantes, lo que les da clara ventaja respecto a los cultivos COP con los que compiten en el caso de un escenario de continuación de la Agenda 2000 (en el que todos los cultivos COP presentan variaciones negativas de precio). Ésta es la razón del importante incremento que experimentan estos cultivos con el escenario de 
la Agenda 2000. Para los escenarios de desacoplamiento, la hipótesis de precios constantes hace a estos cultivos más rentables que algunos cultivos COP de secano, por lo que se incrementa también su superficie, aunque en una menor proporción que con la Agenda 2000. Debido a que el desacoplamiento de las leguminosas es el mismo en los escenarios de desacoplamiento parcial y total, el incremento de su superficie respectiva es mucho mayor en el caso del desacoplamiento total.

En este trabajo hemos formulado la hipótesis de que el precio mundial del algodón se mantenía al mismo nivel del año base. Con esta hipótesis, lógicamente, su superficie cultivada se incrementa en el escenario de la Agenda 2000. En los escenarios de desacoplamiento, el sistema de pago que existía en el año base es sustituido por una ayuda a la superficie cuya parte acoplada representa alrededor de la mitad del montante que le aportaba el sistema de "deficiency payment". Esto tiene como consecuencia la reducción de la superficie cultivada de algodón en estos escenarios.

El fuerte descenso de precios asumido para el arroz, la remolacha azucarera y las patatas da lugar a que sus superficies cultivadas desciendan en todos los escenarios. El descenso es más intenso con la Agenda 2000 debido a los mayores ingresos por hectárea de los cultivos con los que compiten (esencialmente los cultivos COP) en este escenario. Es destacable que, pese al considerable descenso del precio del arroz, su superficie cultivada experimenta una disminución muy pequeña en los escenarios de desacoplamiento, tanto parcial como total. Esto es debido a que en estos escenarios prácticamente se dobla la ayuda directa por ha que tenía este cultivo en el año base. Todos los demás cultivos de este grupo (pimiento para pimentón, espárragos, melón, tomate, pimiento, alcachofa, coliflor, ajo, cebolla y judías verdes) se encuentran esencialmente en las explotaciones tipo 1430 - especializada en hortalizas - en Navarra, Castilla-La Mancha, Murcia y Extremadura. En tales explotaciones, estos cultivos son preponderantes y reemplazan a los cultivos no COP y COP de regadío en el año simulado. Sin embargo, la escala de sustitución es más bien pequeña, como puede deducirse del menor aumento de la superficie dedicada a estos cultivos comparada con el año base, la cual no es mayor del $0,8 \%$ en ninguno de los escenarios simulados.

\section{Resultados para el ganado con diferentes escenarios de política agraria}

En el gráfico 7 se presenta la evolución respecto al año base de las diferentes categorías de ganado consideradas en nuestro modelo con los distintos escenarios de política agraria. 
GRÁFICO 7. VARIACIÓN DEL NÚMERO DE CABEZAS DE LA DIFERENTES CATEGORÍAS DE GANADO

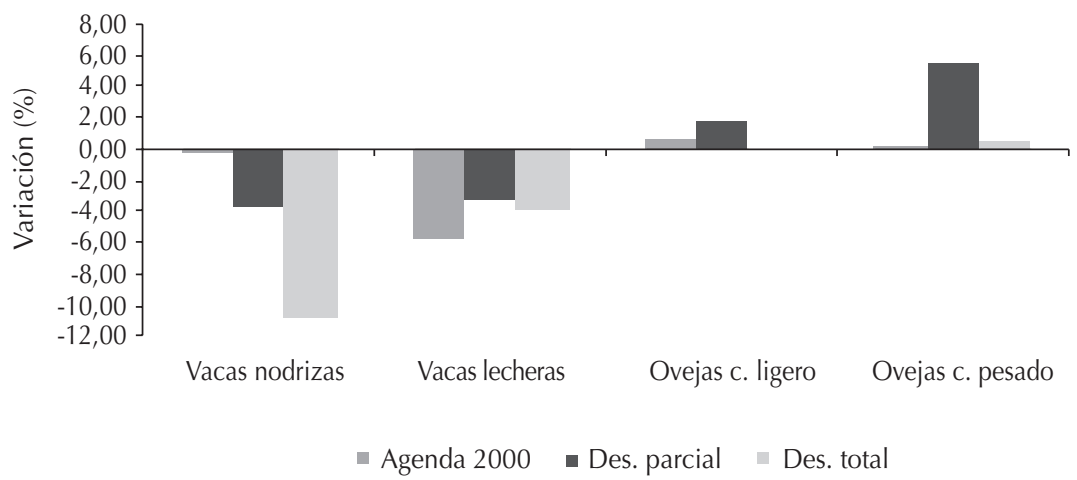

Debido al descenso de precios para la leche y la carne bovina, los tipos de ganado correspondientes disminuyen en todos los escenarios, mientras el ganado ovino cuya tendencia de precios es ascendente, aumenta. Con este punto de partida haremos los comentarios siguientes:

\section{Vacas nodrizas}

El esquema de variación del número de cabezas que muestra el gráfico no es uniforme en las distintas Comunidades Autónomas españolas, presentando importantes variaciones en función de distintos parámetros. Estos parámetros son esencialmente la carga ganadera, el precio de los animales, la proporción de alimentos comprados fuera de la explotación y el precio de esos alimentos.

La suma de ingresos por cabeza procedentes de la venta de ganado 6 de las ayudas acopladas ${ }^{7}$ es función de la carga ganadera. En particular:

- Altas cargas ganaderas que impliquen no recibir ayudas (mayores de 1,9 UGM/ha), hacen que los mayores ingresos se encuentren con el escenario de desacoplamiento total y los menores en el caso de continuación de la Agenda 2000.

- Con cargas ganaderas entre 1,4 y 1,9 UGM / ha en el año base (y entre 1,5 y 1,9 en el año simulado) que facultan a las explotaciones a percibir

6. Tanto mayores cuanto mayor es el grado de desacoplamiento, ya que los precios del bovino disminuyen menos.

7. Tanto menores cuanto mayor es el grado de desacoplamiento. 
primas excluyendo la prima de extensificación, los ingresos más altos se obtienen con el desacoplamiento parcial y los más bajos con el desacoplamiento total

- Para cargas ganaderas bajas que facultan a las explotaciones a percibir primas adicionales, los ingresos más altos se encuentran con la continuación de las medidas de la Agenda 2000, y los más bajos con el escenario de desacoplamiento total.

Las bajas cargas ganaderas se encuentran de forma generalizada en las zonas del centro y sur de España. Es en estas regiones donde, con distintas intensidades, se reproduce el esquema de variaciones que muestra el gráfico 7.

En las regiones del norte de España que constituyen lo que se denomina la "España húmeda", las cargas por ha son mucho más elevadas y muchos tipos de explotaciones se acogen al régimen general de ayudas, mientras otros mantienen una carga ganadera que no les permiten acceder al sistema de ayudas.

En las explotaciones de las Comunidades Autónomas de la "España húmeda" (Galicia, Asturias, Cantabria, País Vasco y Navarra), ricas en pastos, se recurre poco a la compra de alimentación fuera de la explotación y la variación que experimenta este ganado es muy pequeña ${ }^{8}$. Las mayores variaciones se producen con las políticas de desacoplamiento en las otras regiones españolas donde aparece, en general, una compra importante de alimentos fuera de la explotación.

El descenso del precio de los alimentos respecto del año base amortigua sin embargo la disminución del número de cabezas en estas regiones. Otro aspecto observado en algunas explotaciones de la España húmeda es el aumento en el número de vacas nodrizas a expensas del número de vacas lecheras en un intento de maximizar los beneficios del uso de los pastos.

\section{Bovino lechero}

Los parámetros que de una manera general intervienen en las variaciones de este ganado, además del precio de la leche, son el precio de la carne, el precio de los alimentos comprados fuera de la explotación y la proporción

8. Para el conjunto de las explotaciones tipo de estas Comunidades Autónomas, la variación respecto al año base es: $-0,07 \%$ con el escenario de Agenda 2000; 0,15\% con desacoplamiento parcial y $-0,19 \%$ con el escenario de desacoplamiento total. 
consumida por el ganado de estos alimentos. La ayuda acoplada que reciben estas explotaciones en el caso de una política de desacoplamiento parcial, es demasiado pequeña para influir de manera determinante en la evolución de este ganado.

Si se consideran exclusivamente los ingresos acoplados por cabeza procedentes de la venta de leche y de animales jóvenes en todos los tipos de explotación, se obtienen mejores resultados (disminuciones menores respecto a los ingresos del año base) con el escenario de la Agenda 2000 que con los escenarios de desacoplamiento. Sin embargo, el descenso de los precios de los alimentos comprados (en todos los casos estos alimentos representan más del 30\% del total de la materia seca consumida) en los escenarios de desacoplamiento, hace que en muchos casos (en particular en las regiones no pertenecientes a la "España húmeda", donde mayor proporción de alimentos procede de fuera de la explotación), los ingresos directos por cabeza, deducidas las compras de alimento, sean mayores en los escenarios de desacoplamiento. Este hecho explica el patrón de las variaciones según el tipo de escenario de política agraria que encontramos en el gráfico 7.

Debemos señalar, sin embargo, que este patrón no se respeta en algunas Comunidades Autónomas de la España húmeda, como Navarra, con altos rendimientos de leche por vaca, y relativamente pequeñas cantidades de alimentos comprados fuera de la explotación, donde el descenso de los precios de los alimentos no es suficiente para hacer más rentables las vacas lecheras en los escenarios de desacoplamiento que en el escenario de Agenda 2000.

\section{Ovino}

Considerando únicamente los ingresos por cabeza procedentes de la venta de corderos y leche (en el caso de ovejas de cordero ligero), y las ayudas acopladas, el escenario de desacoplamiento parcial con casi el 50\% del total de ayudas acopladas y un aumento importante en el precio de venta de corderos, es el escenario de política agraria más favorable. Las siguientes condiciones más favorables corresponden a la continuación de la Agenda 2000, donde un aumento mucho menor del precio de los corderos se acompaña de la ayuda total acoplada.

Los dos escenarios anteriormente citados ofrecen ingresos acoplados por cabeza de ganado superiores a los del año base, mientras el escenario de desacoplamiento total proporciona unos ingresos acoplados por cabeza inferiores a los del año base, porque la subida del precio de los corderos no compensa totalmente la ausencia de pagos acoplados. 
Cuando para establecer los ingresos acoplados por cabeza se detrae de los mismos el coste de los alimentos comprados, que disminuye en todos los escenarios (aunque más en los escenarios de desacoplamiento), se refuerza la ventaja del escenario de desacoplamiento parcial respecto del año base. Esta mayor ventaja se traduce en incrementos importantes de ovino no lechero en las regiones con alta proporción de alimentos comprados. El menor impacto en las ovejas de cordero ligero es debido a que, como se indicó anteriormente, el precio de la leche de oveja se ha considerado igual al del año base.

Al detraer de los ingresos correspondientes a las ayudas acopladas y a las ventas de corderos y leche, los costes de los alimentos comprados, los ingresos por cabeza de ganado en el escenario de desacoplamiento total son superiores a los del año base y próximos a los obtenidos con el escenario de la Agenda 2000. En algunas Comunidades Autónomas, los ingresos para las ovejas de cordero pesado con el desacoplamiento total son superiores a los que se obtienen con la Agenda 2000, aunque en todas las Comunidades Autónomas se observan pequeñas (o nulas) variaciones en todos los tipos de ganado ovino entre estos dos escenarios de política agraria.

\section{Resultados económicos en diferentes escenarios de politica agraria}

En el gráfico 8 se muestran las variaciones del margen bruto (con y sin ayudas) y la ayuda total (pago por superficie y primas) tras la modulación.

GRÁfico 8. VARIACIÓn DE LOS RESULTADOS ECONÓMICOS RESPECTO AL AÑO BASE

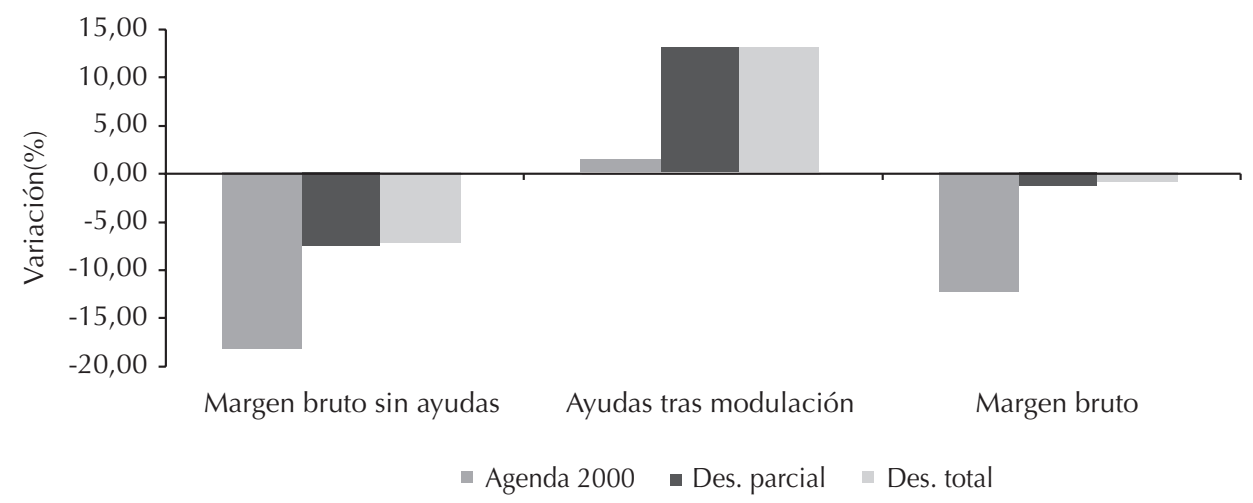


Margen bruto sin ayudas

1. Con el escenario de la Agenda 2000 solamente el ganado ovino experimenta un ligero incremento de precio, mientras los demás productos sufren descensos de precio más o menos importantes ${ }^{9}$. Ésta es la causa de que con este escenario el margen bruto sin ayudas descienda considerablemente respecto al nivel del año base. Este descenso, como puede observarse en el cuadro 7 es prácticamente general en todas las Comunidades Autónomas. La única excepción es Murcia, donde el ganado ovino tiene un peso preponderante en las explotaciones tipo consideradas y donde la alimentación comprada fuera de la explotación representa un alto porcentaje de la alimentación total.

2. Esta variable económica presenta variaciones muy similares en los escenarios de desacoplamiento parcial y total, tanto a nivel global como a nivel de las distintas Comunidades Autónomas.

Comparados con el escenario de la Agenda 2000, los escenarios de desacoplamiento, si se exceptúan los cultivos forrajeros, presentan menos descensos de precios (incluso se observa un pequeño incremento en el caso del trigo) y un aumento considerable del precio de los productos del ganado ovino. Esto explica que, de manera general, el margen bruto sin ayudas sea superior en todas las Comunidades Autónomas, en el caso de los escenarios con desacoplamiento de ayudas ${ }^{10}$.

3. Como ya hemos indicado, en los escenarios de desacoplamiento, sólo el trigo y el ovino presentan incrementos de precios respecto al año base. Esto lleva consigo que en la mayoría de las Comunidades Autónomas el margen bruto sin ayudas sea inferior al del año base. Solamente es superior en La Rioja, Aragón, Castilla-La Mancha, Murcia y Extremadura, que son las Comunidades Autónomas con mayor peso de ganado ovino en las explotaciones.

\section{Ayudas y modulación}

1. Como muestra el cuadro 8 con el escenario de continuación de la Agenda 2000, el nivel de ayudas se mantiene aproximadamente igual al del año base en la mayoría de las Comunidades Autónomas (el caso de Baleares

9. Con la excepción de aquéllos considerados constantes para los productos en los que no se tenía información sobre la evolución de precios. rante.

10. La única excepción es Cataluña, donde la alfalfa vendida juega un papel preponde- 
no es significativo porque el montante de ayudas, con la Agenda 2000, es muy pequeño para las explotaciones consideradas), lo que explica la pequeña variación a nivel global. La variación positiva más importante tiene lugar en Andalucía debido, fundamentalmente, al incremento de la superficie de algodón.

2. El aumento de las ayudas que se observa, a nivel global, en los escenarios de desacoplamiento es debido en gran parte a que, en ellos, se consideran ayudas acopladas o desacopladas, para algunos productos, superiores a las que existían en el año base (caso del arroz) o que no existían en dicho año (leche y remolacha azucarera). Esto explica, por otra parte, el considerable aumento de ayudas que se observa en Baleares y en Valencia. En Baleares, la única actividad con una cierta importancia con derecho a ayudas en los escenarios de desacoplamiento es la del bovino lechero que, como ya hemos indicado, no recibía ninguna ayuda en el año base. En el caso de Valencia se debe al fuerte incremento de ayuda que experimenta el cultivo del arroz. Tras estas Comunidades Autónomas, también se observan incrementos importantes de las ayudas en las Comunidades Autónomas del norte de España (Galicia, Asturias y Cantabria), donde la producción de leche es la actividad preponderante. Destacaremos, finalmente, que en algunas Comunidades Autónomas, se producen disminuciones de ayudas, siendo el caso más notable el de Madrid, debido a que la hipótesis retenida en este trabajo llevaría a una disminución importante del número de vacas nodrizas, y a que en esta Comunidad Autónoma no se encuentran los productos que han experimentado un aumento de ayudas.

3. La reducción de las ayudas por modulación representa a nivel global un poco más del 2\% del total de ayudas. Esta proporción difiere entre las distintas Comunidades Autónomas, y oscila entre el 1\% de Cantabria hasta un poco más del 3\% en Andalucía considerando en las distintas comunidades únicamente aquellas explotaciones tipo con dimensión suficiente para tener una reducción de ayudas.

\section{Margen bruto}

1. Este indicador económico (la suma del margen bruto sin ayudas, más las ayudas), muestra que las políticas de desacoplamiento producen mejores resultados que la continuación de la Agenda 2000 en la práctica totalidad de las Comunidades Autónomas (cuadro 9 y gráfico 8), aunque en la mayoría de los casos se constatan variaciones negativas en términos reales. 
2. En la mayoría de las Comunidades Autónomas, el margen bruto bajo el escenario de desacoplamiento total es superior al obtenido con el desacoplamiento parcial. En las Comunidades Autónomas donde esto no sucede, la causa es que el paso de un desacoplamiento parcial a uno total implica una pérdida importante de actividad agraria, tal como sucede en Madrid ${ }^{11}$.

CuAdro 7. Margen bruto sin ayudas (\% Variación Respecto al aÑo base)

Agenda 2000 Desacoplamiento parcial Desacoplamiento total

\begin{tabular}{lrrr}
\hline 01. Galicia & $-22,35$ & $-17,09$ & $-16,23$ \\
02. Asturias & $-19,93$ & $-17,76$ & $-16,35$ \\
03. Cantabria & $-22,82$ & $-21,4$ & $-20,41$ \\
04. País Vasco & $-18,28$ & $-15,09$ & $-13,56$ \\
05. Navarra & $-13,53$ & $-3,21$ & $-1,06$ \\
06. La Rioja & $-28,29$ & 5,01 & 6,22 \\
07. Aragón & $-18,87$ & 1,67 & 3,31 \\
08. Cataluña & $-31,38$ & $-33,86$ & $-35,13$ \\
09. Islas Baleares & $-36,23$ & $-17,58$ & $-17,62$ \\
10. Castilla-León & $-18,22$ & $-6,47$ & $-7,12$ \\
11. Madrid & $-13,47$ & $-1,74$ & $-2,51$ \\
12. Castilla-La Mancha & $-7,77$ & 6,64 & 7,04 \\
13. Comunidad Valenciana & $-60,77$ & $-59,58$ & $-59,51$ \\
14. Murcia & 12,52 & 41,09 & 42,11 \\
15. Extremadura & $-10,01$ & 10,76 & 13,48 \\
16. Andalucía & $-25,88$ & $-12,45$ & $-12,99$ \\
00. España & $-18,41$ & $-7,66$ & $-7,22$ \\
& & & \\
\hline
\end{tabular}

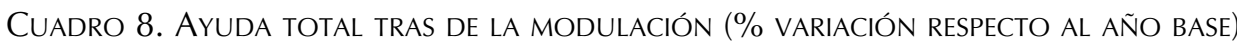

Agenda 2000 Desacoplamiento parcial Desacoplamiento total

\begin{tabular}{llrc}
\hline 01. Galicia & 0,27 & 294,99 & 294,02 \\
02. Asturias & 0 & 183,03 & 183,08 \\
03. Cantabria & 0 & 189,35 & 190,84 \\
04. País Vasco & 0 & 50,48 & 50,76 \\
05. Navarra & 0,88 & 8,65 & 8,69 \\
06. La Rioja & 0,71 & 33,38 & 30,79 \\
07. Aragón & 1,41 & 0,91 & $-1,21$ \\
08. Cataluña & 2,06 & 16 & 17,98 \\
09. Islas Baleares & 65,59 & 7292,95 & 7282,8 \\
10. Castilla-León & 1,42 & 11,62 & 11,36 \\
11. Madrid & $-0,02$ & $-16,07$ & $-39,77$ \\
\hline
\end{tabular}

11. La misma situación se da en La Rioja, Aragón, Murcia, Cataluña, Islas Baleares y Castilla-León, aunque aquí la diferencia en los márgenes brutos bajo los dos escenarios es pequeña. 
572 • LUCINIO JÚDEZ, M. a DEL ROSARIO DE ANDRÉS, MIGUEL IBÁÑEZ, ELVIRA URZAINQUI Y JOSÉ LUIS MIGUEL

Cuadro 8. Ayuda tOtal tRas de la mODULACIÓn (\% VARIACIÓn RESPECTO AL AÑO BASE) (CONT.)

Agenda 2000 Desacoplamiento parcial Desacoplamiento total

\begin{tabular}{lrrr}
\hline 12. Castilla-La Mancha & $-0,05$ & $-1,59$ & $-1,78$ \\
13. Comunidad Valenciana & 0,37 & 344,37 & 344,45 \\
14. Murcia & 0,36 & 4,67 & 0,18 \\
15. Extremadura & 0,33 & $-1,96$ & 0,21 \\
16. Andalucía & 2,97 & 9,66 & 10,56 \\
00. España & 1,39 & 13,04 & 12,92 \\
\hline
\end{tabular}

Cuadro 9. Margen bruto (\% VARIación RESPECto al año baSe)

Agenda $2000 \quad$ Desacoplamiento parcial Desacoplamiento total

\begin{tabular}{lccc}
\hline 01. Galicia & $-21,47$ & $-4,94$ & $-4,15$ \\
02. Asturias & $-18,9$ & $-7,37$ & $-6,03$ \\
03. Cantabria & $-21,44$ & $-8,67$ & $-7,64$ \\
04. País Vasco & $-15,52$ & $-5,19$ & $-3,85$ \\
05. Navarra & $-9,56$ & 0,06 & 1,62 \\
06. La Rioja & $-18,69$ & 14,41 & 14,36 \\
07. Aragón & $-9,88$ & 1,33 & 1,31 \\
08. Cataluña & $-22,93$ & $-21,26$ & $-21,71$ \\
09. Islas Baleares & $-36,01$ & $-1,85$ & $-1,91$ \\
10. Castilla-León & $-11,89$ & $-0,64$ & $-1,17$ \\
11. Madrid & $-8,6$ & $-6,93$ & $-16,01$ \\
12. Castilla-La Mancha & $-5,07$ & 3,76 & 3,96 \\
13. Comunidad Valenciana & $-52,97$ & $-8,06$ & $-7,98$ \\
14. Murcia & 9,58 & 32,28 & 31,96 \\
15. Extremadura & $-5,82$ & 5,61 & 8,11 \\
16. Andalucía & $-11,2$ & $-1,2$ & -1 \\
00. España & $-12,26$ & $-1,23$ & $-0,96$ \\
\hline
\end{tabular}

\section{COMENTARIOS FINALES}

En las páginas anteriores hemos hecho un análisis detallado, a partir de los resultados de PROMAPA, de los efectos de políticas agrarias, con varios grados de desacoplamiento de las ayudas, sobre la agricultura española.

Una conclusión de tipo general es que bajo los escenarios de desacoplamiento parcial o total la actividad agraria disminuye más (se dejan más hectáreas sin cultivar y se pierden más cabezas de ganado) que con el escenario de continuación de la Agenda 2000. Sin embargo, con este último escenario las ganancias de los agricultores son menores. La ventaja de los escenarios de desacoplamiento, respecto a la renta de los agricultores, es debida por un lado 
a previsiones de precios más elevados y, por otro, a que con estos escenarios aumentan las ayudas para algunos productos y aparecen nuevas ayudas que no existían en la Agenda 2000, previstas para compensar la reducción de precios garantizados que impuso la Reforma a Medio Plazo de la PAC.

El fuerte incremento en términos reales, del precio previsto por ESIM entre 2002 y 2013 para el ovino, junto con el descenso de precios estimados para la práctica totalidad del resto de productos, en los escenarios de desacoplamiento lleva consigo que las comunidades autónomas con mayor peso en ovino (La Rioja, Aragón, Castilla-La Mancha, Murcia y Extremadura) sean las únicas junto con Navarra en las que se observa con estos escenarios un incremento del margen bruto respecto al existente en el año base.

También cabe destacar que las políticas de desacoplamiento, con las hipótesis de precios retenidas en este estudio, conducen a la disminución de la cabaña de bovino para cría. Esta disminución sin embargo no es homogénea en toda España, presentando muy pequeñas variaciones respecto al año base en las comunidades autónomas pertenecientes a la España húmeda (Galicia, Asturias, Cantabria, País Vasco y Navarra) debido a que sus explotaciones poseen pastos abundantes y recurren poco a la compra de alimentos fuera de la explotación.

Independientemente de los resultados hemos de señalar que el modeIo PROMAPA es un instrumento flexible y de manejo relativamente sencillo concebido para ser una herramienta de ayuda a la reflexión sobre las consecuencias que pueden esperarse de la implantación de nuevas medidas de política agraria sobre el sector agrario español tanto a nivel global como a nivel de distintas Comunidades Autónomas y de diferentes Orientaciones Técnico Económicas. En las páginas anteriores hemos ilustrado la utilización del modelo para analizar los efectos de las medidas asociadas a la nueva reforma de la PAC asumiendo los resultados sobre variaciones de precios del modelo europeo de simulación (ESIM).

Recientemente la extensión de cultivos agro-energéticos y el aumento de la demanda de cereales a nivel mundial han conducido a un incremento espectacular de los precios de las materias primas. Estos hechos ponen en evidencia el interés de comparar los resultados presentados en este trabajo, que fue una de las bases del informe presentado a la UE en 2007 en el contexto del Proyecto europeo GENEDEC, con los que se obtengan con una predicción de precios más ajustada a las actuales variaciones de precios introduciendo además las muy recientes modificaciones de medidas de política agraria.

Recibido 23.06.08

Aceptado 05.10.08 


\section{BIBLIOGRAFÍA}

Adenáuer, M.; Britz, W.; Gocht, A.; Gömann, H.; Cristoiu, A. y Ratinger, T. (2006): ModeIling impacts of decoupled premiums: building-up a farm type layer within the EU-wide regionalised CAPRI model, Selected paper presented at the $93^{\text {rd }}$ EAAE Seminar, Praga, 22-23 septiembre.

Arfini, F. y Paris, Q. (1995): “A positive mathematical programming model for regional analysis of agricultural policies", en Sotte, E., The Regional Dimension in Agricultural Economics and Policies, EAAE, Proceedings of the $40^{\text {th }}$ Seminar, 26-28 junio, pp. 17-35.

Arriaza, M. y Gómez-Limón, J. A. (2003): "Comparative performance of selected mathematical programming models", Agricultural Systems, no 77, pp. 155-171.

Barkaoui, A. y Butault, J. P. (1999): Positive Mathematical Programming and Cereals and Oilseeds Supply within EU under Agenda 2000, Paper presented at the $9^{\text {th }}$ European Congress of Agricultural Economics, Varsovia, agosto.

Barkaoui, A. y Butault, J. P. (2003): Révision à mi-parcours de la PAC : simulations de l'effect du découplage sur l'offre dans les régions françaises, Document de travail, INRA-ESR Nancy.

Blanco, A. e Iglesias, E. (2005): Modelling New EU Agricultural Policies: Global Guidelines, Local Strategies, Selected paper presented at the $89^{\text {th }}$ EAAE Seminar, 3-5 febrero, Parma.

Buysse, J.; Fernagut, B.; Harmignie, O.; Henry De Frahan, B.; Lauwers, L.; Polomé, P.; Van Huylenbroeck, G. y Van Meensel, J. (2004): Modelling the impact of sugar reform on Belgian Agriculture, Selected paper presented at the International Conference on Policy Modelling, París, 30 junio-2 julio.

Buysse, J. y Van Huylenbroeck, G. (2005): Impact of alternative implementations of the Agenda 2000 Mid Term Review, Selected Paper presented at the $11^{\text {th }}$ Congress of the EAAE, Copenhague, Dinamarca, agosto 24-27.

Buysse, J.; Van Huylenbroeck, G. y Lauwers, L. (2007): “Normative, positive and econometric mathematical programming as tools for incorporation of multifunctionality in agricultural policy modelling", Agriculture, Ecosystems \& Environment, 120, pp. 70-78.

CAPRI (2000): Common Agricultural Policy Regional Impact (Fair3-CT96-1849), Final Consolidated Report, Institute for Agricultural Policy, University of Bonn, Department of Economics, University College Galway, Institut Agronomique Meditérranéen de Montpellier, Departamento di Economia, Sociologia y Politica Agraria, Universidad Politécnica de Valencia, Università degli Studi di Bologna, Dipartamento di Protezione e Valorizzazione Agro-Alimentare (DIPROVAL), Sezione Economía.

Gohin, A. y Chantreuil, F. (1999): "La programmation mathématique positive dans les modèles d'exploitation agricole: principes et importance du calibrage", Cahiers d'Économie et Sociologie Rurales, $\mathrm{n}^{\circ}$ 52, pp. 59-78.

Hazell, B. R. y Norton, R. D. (1986): Mathematical Programming for Economic Analysis in Agriculture, McMillan, Nueva York.

Heckelei, T. y Britz, W. (1999): Maximum entropy specification of PMP, CAPRI Working Paper 08199, en: http://www.agp.uni-bonn.de/agpo/rsch/capri/wrkpap-e.htm

Heckelei, T. y Wolff, H. (2003): "Estimation of Constrained Optimisation Models for Agricultural Supply Analysis Based on Generalised Maximum Entropy", European Review of Agricultural Economics, $\mathrm{n}^{\circ}$ 30(1), pp. 27-50.

Heckelei, T. y Britz, W. (2005): Models Based on Positive Mathematical Programming: State of the Art and Futher Extensions, Plenary paper presented at the 89 ${ }^{\text {th }}$ EAAE Seminar, 3-5 febrero, Parma, pp. 48-73.

Helming, J. F. M.; Peeters, L. y Veendendaal, P. J. J. (2001): “Assessing the Consequences of Environmental Policy Scenarios in Flemish Agriculture", en Heckelei, T.; Witzke, H. P. 
y Henrichsmeyer, W. (eds.), Agricultural Sector Modelling and Policy Information Systems, Vank Verlag, Kiel.

Henry de Frahan, B. (2005): PMP, Extensions and Alternative Methods: Introductory Review of the State of the Art, Selected Paper presented at the $11^{\text {th }}$ Congress of the EAAE, Copenhague, Dinamarca, agosto 24-27.

Howitt, R. E. (1995): "Positive Mathematical Programming", American Journal of Agricultural Economics, n $77(2)$, pp. 329-342.

Ibáñez J. y Pérez C. (1999): "Impactos de la Reforma de la PAC de 1992 sobre el subsector agrícola español", Revista de Estudios Agrosociales y Pesqueros, n 185, pp. 9-30.

Jayet, P. A.; Marzochi, E.; Hofstetter, A. y Donati, M. (2000): Modular approach of agricultural supply modelling through linear programming, EUROTOOLS, Working Paper, Series $\mathrm{n}^{\circ} 26$.

Jayet, P.A. (ed.) (2007): Policy Recommmendation, Work Package 7, Deliverable D10, GENEDEC, Project 502184.

Júdez, L.; Martínez, S. y Fuentes-Pila, J. (1998): Positive Mathematical Programming Revisited, Trabajo presentado al meeting de Madrid, 16-17 de octubre sobre EUROTOOLS (Proyecto FAIR 5-CT97-3403).

Júdez, L.; Chaya, C.; Martínez, S. y González, A. A. (2001): “Effects of the Measures Envisaged in 'Agenda 2000' on Arable Crop Producers and Beef and Veal Producers: An Application of Positive Mathematical Programming to Representative Farms of a Spanish Region", Agricultural Systems, n 67, pp. 121-138.

Júdez, L.; De Miguel, J. M.; Piniés, M.; Legorburu, I. G. y Miguel, J. L. (2004): Effects of Mid-Term review measures on some representative Spanish farm: an application of the PROMAPA model, Selected paper presented 80 th EAAE Seminar, Ghent, 24-26 septiembre.

Júdez, L.; De Andrés, R.; Ibáñez, M.; De Miguel, J. M. y Urzainqui, E. (2005): The PROMAPA.G Model, Document of GENEDEC Project, 31 marzo.

Kuepker, B. y Kleinhanss, W. (2006): Comparative Assessment of National and Alternative Decoupling Schemes in EU Member States, Selected paper presented at the 93 rd EAAE Seminar, Praga 22-23 septiembre.

Offermann, F.; Kleinhanss, W.; Huettel, S. y Kuepker, B. (2005): "Assessing the 2003 CAP reform impacts on German Agriculture using the farm group model FARMIS", en Arfini, F. (ed.), Modelling agricultural policies: state of the art and new challenges, Proceedings of the $89^{\text {th }}$ EAAE Seminar, Parma.

Osterburg, B.; Offermann, F. y Kleinhanss, W. (2001): "A sector consistent farm group model for German agriculture", en Heckelei, T.; Witzke, H. P. y Henrichsmeyer, W. (eds.), Agricultural Sector Modelling and Policy Information Systems, Kiel, Wissenschaftsverlag, Vauk.

Paris, Q. y Howitt, R. E. (1998): "An Analysis of Ill-posed Production Problems Using Maximum Entropy", American Journal of Agricultural Economics, n 80(1), pp. 124138.

Paris, Q.; Montresor, E.; Arfini, F. y Mazzocchi, M. (2000): An Integrated Multi-Phase Model for Evaluating Agricultural Policies Through Positive Information, en Heckelei, T.; Witzke, H. P. y Henrichsmeyer, W. (eds.), Agricultural Sector Modelling and Policy Information Systems, Proceedings of the $65^{\text {th }}$ EAAE Seminar, marzo 29-31, Bonn University, Vauk Verlag Kiel, pp. 100-110.

Röhm, O. y Dabbert, S. (1999): "Modelling regional production and Income Effects", en Huylenbroeck, G. y Witby, M. (eds.), Countryside Stewardship, Farmers, Policies and Markets, Pergamon, Ámsterdam.

Röhm, O. y Dabbert, S. (2003): "Integrating Agri-Environmental Programs into Regional Production Models: An Extension of Positive Mathematical Programming", American Journal of Agricultural Economics, $\mathrm{n}^{\circ}$ 85(1), pp. 254-265. 


\title{
Resumen
}

En este trabajo se analiza el impacto sobre el sector agrario español de las medidas de la Política Agraria Común de desacoplamiento de ayudas directas a las superficies sembradas de distintos cultivos y a las cabezas de ganado.

El análisis se hace en base a los datos de 2002 de la Red Contable Nacional Agraria y a los resultados simulados para 2013 con el modelo de programación matemática PROMAPA, utilizando la predicción de variacion de precios entre 2002 y 2013 del modelo europeo de simulación ESIM.

Los resultados obtenidos permiten comparar los cambios en la distribución de cultivos y de distintas categorías de ganado entre el año base 2002 y el año simulado, 2013, según se siga una política de desacoplamiento parcial, como la implantada en España en 2006, o una política de desacoplamiento total sobre la que se está reflexionando actualmente.

En este trabajo se comparan, por otra parte, los cambios que se observan con los dos tipos de desacoplamiento con los que se obtendrían en el caso de proseguir las medidas vigentes en 2002 en el contexto de la denomina Agenda 2000.

Palabras clave: Política Agraria Comunitaria; modelos de explotaciones representativas; sector agrario español; distribución de cultivos y ganado.

\begin{abstract}
This paper analyses the impact on the Spanish agricultural sector of the CAP decoupling measures of direct payments for different crop areas and livestock.

The study is based in the 2002 data from the Spanish Farm Accountancy Data Network (FADN) and the simulated results are obtained with the mathematical programming model PROMAPA. The predicted changes in prices between the years 2002 and 2013 were those provided by the European Simulation Model (ESIM).

The results obtained permit the comparison of the variation in the crop distribution and of the different types of livestock between the base year 2002 and the simulated year 2013 in the event of the implementation of a partial decoupling policy —as adopted in Spain in 2006or a full decoupling policy which is under consideration at the moment.

This paper also compares the variations which appear in the two types of decoupling with those that would be obtained in the even of a continuation of the Agenda 2000 measures.
\end{abstract}

Key words: Common Agricultural Policy; representative farm models; Spanish agricultural sector; crop and livestock distribution. 